\title{
Reduction of Multiple Harmonic Sums and Harmonic Polylogarithms
}

\author{
J. Blümlein ${ }^{\mathrm{a}}$ \\ ${ }^{\text {a} D E S Y, ~ D e u t s c h e s ~ E l e k t r o n e n ~ S y n c h r o t r o n, ~ D E S Y, ~ P l a t a n e n a l l e e ~ 6, ~ D-15738 ~ Z e u t h e n, ~ G e r m a n y ~}$
}

The alternating and non-alternating harmonic sums and other algebraic objects of the same equivalence class are connected by algebraic relations which are induced by the product of these quantities and which depend on their index calss rather than on their value. We show how to find a basis of the associated algebra. The length of the basis $l$ is found to be $\leq 1 / d$, where $d$ is the depth of the sums considered and is given by the 2nd WITT formula. It can be also determined counting the LYNDON words of the respective index set. The relations derived can be used to simplify results of higher order calculations in QED and QCD.

\section{INTRODUCTION}

Multiple nested alternating and non-alternating harmonic sums $S_{a_{1}, \ldots, a_{n}}(N)[1-3]$ emerge in perturbative higher order calculations within QED and QCD for massless fermions,

$$
\begin{aligned}
S_{a_{1}, \ldots, a_{n}}(N)= & \sum_{k_{1}=1}^{N} \sum_{k_{2}=1}^{k_{1}} \ldots \sum_{k_{n}=1}^{k_{n-1}} \frac{\operatorname{sign}\left(a_{1}\right)^{k_{1}}}{k_{1}^{\left|a_{1}\right|}} \\
& \ldots \frac{\operatorname{sign}\left(a_{n}\right)^{k_{n}}}{k_{n}^{\left|a_{n}\right|}} .
\end{aligned}
$$

Here, $a_{k}$ are positive or negative integers and $N$ is a positive even or odd integer depending on the observable under consideration. One calls $n$ the depth and $\sum_{k=1}^{n}\left|a_{k}\right|$ the weight of a harmonic sum. Harmonic sums are associated to MELLin transforms of real functions or SCHWARTZ-distributions $f(x) \in \mathcal{S}^{\prime}[0,1][4]$

$$
S_{a_{1}, \ldots, a_{n}}(N)=\int_{0}^{1} d x x^{N} f_{a_{1}, \ldots, a_{n}}(x)
$$

which emerge in field theoretic calculations. Finite harmonic sums are related to harmonic polylogarithms $H_{b_{1}, \ldots, b_{n}}(x)$ [5]. Their $1 /(1 \pm$ $x$ )-weighted MELLIN transform yields harmonic sums. The inverse MeLLIN transform relates the harmonic sums to functions of NIELSEN integrals [6] of the variable $x$ at least for all sums of weight $w \leq 4$ as shown in [3], and associated generalizations for higher weight. NIELSEN integrals are a generalization of the usual polylog- arithms [7]. In the limit $N \rightarrow \infty$ the convergent multiple harmonic sums, i.e. those where $a_{1} \neq 1$, yield (multiple) Zeta-values $\zeta_{a_{1}, \ldots, a_{n}}$, which are also called EULER-ZAGIER sums [8]. A generalization of both harmonic polylogarithms and the EULER-ZAGIER sums are the nested $Z_{-}$ sums [9], which form a HopF algebra [10,11] and are related to Goncharov's multiple polylogarithms [12]. For a recent review see [13].

Higher order calculations in massless filed theories are either performed in MELLIN- $N$ space referring to harmonic sums or in the space of the momentum fractions $x$ representing the results in terms of NIELSEN-type integrals. The principal complexity is determined by the amount of possible terms contributing. In the case of the $2-$ loop coefficient functions in momentum fraction space [14] 77 different functions occurred, cf. [3]. This number compares to the amount of all possible different nested harmonic sums up to weight $w=4,80=3^{w}-1$. For the 3 -loop anomalous dimensions [15] one expects the contribution of a wide class of the $w=5$ harmonic sums and for the 3-loop coefficient functions of the $w=6$ harmonic sums, which means 242 or 728 sums, respectively. These sums are not independent but connected by different kind of relations. In the present paper we summarize a first class of relations recently being discussed in Ref. [16], the so-called algebraic relations. ${ }^{1}$ It turns out that

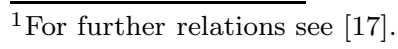


these relations emerge from the index-structure and the multiplication relation of the objects considered and are widely independent of other properties of the harmonic sums. In this way an equivalence class of even more objects is defined having the same properties or can be found as special cases thereof. One example is the set of the harmonic polylogarithms [5].

To obtain manageable expressions it is of importance to apply all these relations through which the number of basic functions to be referred to is considerably reduced. Experience shows that the MELLIN space representation yields simpler expressions in general [18], which would not be seen easily working in $x$ space. To obtain as simple as possible expressions it is of special importance because of the fact that data-analyses require compact results in $x$-space, which can be obtained using analytic continuations for the basic sums [19] and performing a single numeric MELLin inversion [20] for the whole problem. Since the evaluation of precise analytic continuations needs special effort any possible reduction carried out before is of help.

\section{ALGEBRAIC RELATIONS}

The product of two finite harmonic sums (1) yields

$$
\begin{aligned}
& S_{a_{1}, \ldots, a_{n}}(N) \cdot S_{b_{1}, \ldots, b_{m}}(N) \\
& =\sum_{l_{1}=1}^{N} \frac{\operatorname{sign}\left(a_{1}\right)^{l_{1}}}{l_{1}^{\left|a_{1}\right|}} S_{a_{2}, \ldots, a_{n}}\left(l_{1}\right) S_{b_{1}, \ldots, b_{m}}\left(l_{1}\right) \\
& +\sum_{l_{2}=1}^{N} \frac{\operatorname{sign}\left(b_{1}\right)^{l_{2}}}{l_{2}^{\left|b_{1}\right|}} S_{a_{1}, \ldots, a_{n}}\left(l_{2}\right) S_{b_{2}, \ldots, b_{m}}\left(l_{2}\right) \\
& -\sum_{l=1}^{N} \frac{\left[\operatorname{sign}\left(a_{1}\right) \operatorname{sign}\left(b_{1}\right)\right]^{l}}{l^{\left|a_{1}\right|+\left|b_{1}\right|}} S_{a_{2}, \ldots, a_{n}}(l) S_{b_{2}, \ldots, b_{m}}(l) .
\end{aligned}
$$

We introduce the shuffle product $\amalg$ of a single and a general finite harmonic sum

$$
\begin{aligned}
& S_{a_{1}}(N) \sqcup S_{b_{1}, \ldots, b_{m}}(N)=S_{a_{1}, b_{1}, \ldots, b_{m}}(N) \\
& +S_{b_{1}, a_{1}, b_{2}, \ldots, b_{m}}(N)+\ldots+S_{b_{1}, b_{2}, \ldots, b_{m}, a_{1}}(N)
\end{aligned}
$$

which is a linear combination of the sums of depth $m+1$. The shuffle product of two harmonic sums of depth $n$ and $m, S_{a_{1}, \ldots, a_{n}}(N)$ and $S_{b_{1}, \ldots, b_{m}}(N)$, is the sum of all harmonic sums of depth $m+n$ in the index set of which $a_{i}$ occurs left of $a_{j}$ for $i<j$ and likewise for $b_{k}$ and $b_{l}$ for $k<l$. As an example the shuffle product of two threefold harmonic sums is given by

$$
\begin{aligned}
& S_{a_{1}, a_{2}, a_{3}}(N) \sqcup S_{a_{4}, a_{5}, a_{6}}(N)= \\
& S_{a_{1}, a_{2}, a_{3}, a_{4}, a_{5}, a_{6}}(N)+S_{a_{1}, a_{2}, a_{4}, a_{3}, a_{5}, a_{6}}(N) \\
& +S_{a_{1}, a_{2}, a_{4}, a_{5}, a_{3}, a_{6}}(N)+S_{a_{1}, a_{2}, a_{4}, a_{5}, a_{6}, a_{3}}(N) \\
& +S_{a_{1}, a_{4}, a_{2}, a_{3}, a_{5}, a_{6}}(N)+S_{a_{1}, a_{4}, a_{2}, a_{5}, a_{3}, a_{6}}(N) \\
& +S_{a_{1}, a_{4}, a_{2}, a_{5}, a_{6}, a_{3}}(N)+S_{a_{1}, a_{4}, a_{5}, a_{6}, a_{2}, a_{3}}(N) \\
& +S_{a_{1}, a_{4}, a_{5}, a_{2}, a_{6}, a_{3}}(N)+S_{a_{1}, a_{4}, a_{5}, a_{2}, a_{3}, a_{6}}(N) \\
& +S_{a_{4}, a_{5}, a_{6}, a_{1}, a_{2}, a_{3}}(N)+S_{a_{4}, a_{5}, a_{1}, a_{6}, a_{2}, a_{3}}(N) \\
& +S_{a_{4}, a_{5}, a_{1}, a_{2}, a_{6}, a_{3}}(N)+S_{a_{4}, a_{5}, a_{1}, a_{2}, a_{3}, a_{6}}(N) \\
& +S_{a_{4}, a_{1}, a_{5}, a_{6}, a_{2}, a_{3}}(N)+S_{a_{4}, a_{1}, a_{5}, a_{2}, a_{6}, a_{3}}(N) \\
& +S_{a_{4}, a_{1}, a_{5}, a_{2}, a_{3}, a_{6}}(N)+S_{a_{4}, a_{1}, a_{2}, a_{3}, a_{5}, a_{6}}(N) \\
& +S_{a_{4}, a_{1}, a_{2}, a_{5}, a_{3}, a_{6}}(N)+S_{a_{4}, a_{1}, a_{2}, a_{5}, a_{6}, a_{3}}(N)
\end{aligned}
$$

Finally one establishes a system of linear equations in which the linear elements of the shuffle products form the variables and a polynomial out of harmonic sums of lower depth forms the inhomogeneity. We furthermore consider all index permutations. This system contains all algebraic relations. In Ref. [16] all solutions for harmonic sums up to depth $d=6$ were given. This complies to the level of sophistication needed to reduce the corresponding relations which emerge for massless 3-loop coefficient functions.

\section{NUMBER OF ALGEBRAICALLY IN- DEPENDENT HARMONIC SUMS}

Let us consider the index set of a harmonic sum of depth $d$. One may consider this set as a word $w$ or a non-commutative product of letters of an ordered alphabet $\mathfrak{A}=\{a, b, c, d, \ldots\}$. Any word can be decomposed into three parts

$$
w=p x s,
$$

a prefix $p$, a suffix $s$, and the remainder part $x$. Among all words $w$ the LYNDON words, cf. e.g. [21], are those being smaller than any of its suffixes.

According to a Theorem by RADFORD [22] the shuffle algebra discussed above is freely generated 
by the LYNDON words, i.e. the length of its basis is given by the number of LYNDON words. We would like to count the number of LYNDON words for index sets, where the same letters can emerge repeatedly. The corresponding relation is due to Wiтt [23] and will be called 2nd WiTT formula

$$
l_{n}\left(n_{1}, \ldots, n_{q}\right)=\frac{1}{n} \sum_{d \mid n_{i}} \mu(d) \frac{\left(\frac{n}{d}\right) !}{\left(\frac{n_{1}}{d}\right) ! \ldots\left(\frac{n_{q}}{d}\right) !}
$$

with $n=\sum_{k=1}^{q} n_{k}$. Here $\mu(d)$ denotes the MöBIUs function. One may derive $l_{n}\left(n_{1}, \ldots, l_{q}\right)$ using the generating functional

$\frac{1}{1-x_{1}-\ldots-x_{n_{q}}}=\prod_{n=1}^{\infty}\left(\frac{1}{1-\sum_{k=1}^{q} x_{k}^{d_{k}}}\right)^{l_{n}\left(n_{i}\right)}$

Note that (7) is related to the GAUSS-WITT relation mentioned by HofFMAN [24] for the number of basic multiple Zeta-values of weight $w$ for $\forall n_{i}>0$ if all cases for fixed weight are summed over. An even more strict relation in the inclusive case has been conjectured by ZAGIER [8] and BroAdhurst and Kreimer [25] in the case of multiple Zeta-values and verified up to $w=12$.

Let us come back to Eq. (7). We can draw some immediate conclusions out of this relation. If the numbers $n_{i}$ have no common divisor larger than 1 , the number of the basis elements compared to the number of all objects equals $1 / d$, where $d$ denotes the depth of the index set. In case of common divisors larger than 1 we checked that the basis is always shorter for all depths up to $d=10$, see [16].

\begin{tabular}{||r||r|r|l||}
\hline \hline Weight & \# Sums & \# Basic Sums & Fraction \\
\hline 1 & 2 & 0 & 0.0 \\
2 & 8 & 1 & 0.1250 \\
3 & 26 & 7 & 0.2692 \\
4 & 80 & 23 & 0.2875 \\
5 & 242 & 69 & 0.2851 \\
6 & 728 & 183 & 0.2513 \\
\hline \hline
\end{tabular}

\section{CONCLUSIONS}

The product of finite alternating or nonalternating harmonic sums is given by the shuffle product of harmonic sums and polynomials of harmonic sums of lower depth. These representations imply algebraic relations between the harmonic sums. If one considers all harmonic sums associated to an index set $\left\{a_{1}, \ldots, a_{k}\right\}$ one may express these sums by a number of basic sums. It turns out that this number is given by the 2 nd WITT formula which counts the number of LYNDON words corresponding to the respective index set. The set of these LYNDON words generates in this sense all harmonic sums of this class freely. By solving the corresponding linear equations we derived the explicit representation of all harmonic sums up to depth $d=6$ without specifying the indices numerically and gave all expression which are structurally needed to express the sums up to weight $w=6$. The counting relations for the basis of the finite harmonic sums were given up to depth $d=10$. The relations derived hold likewise for other mathematical objects obeying the same multiplication relation or a simpler one, which is being contained, as that for harmonic polylogarithms. This is due to the fact that the relations derived depend on the index set and the multiplication relation but on no further properties of the objects considered.

The ratio of the number of basic sums for a given index set and the number of all sums is mainly determined by the depth $d$ rather than the weight of the respective sums, due to the prefactor $1 / d$ in the WITT formula. Modifications occur due to common non-trivial divisors of the numbers of individual indices in the set being considered. Up to $d=10$ we showed that the fraction of basic sums is always $\leq 1 / d$ compared to all sums. The use of these algebraic relations leads to a considerable reduction in the set of functions needed to express the results of higher order calculations in massless QED and QCD and related subjects. For practical applications such as the description of the QCD scaling violation of the structure functions in deeply inelastic scattering the harmonic sums occurring in the MELLIN $N$ space calculation have to be translated to $x$-space by the inverse MELLIN transform. For this reason the respective harmonic sums have to be analytically continued in the argument $N$ to complex values, which requires a high effort using numerical procedures. It is therefore recommended to 
use as many as possible relations between the $N$ space objects before to perform the last step only for the reduced set.

Acknowledgment. This paper was supported in part by DFG Sonderforschungsbereich Transregio 9, Computergestützte Theoretische Physik.

\section{REFERENCES}

1. A. Gonzalez-Arroyo, C. Lopez, and F.J. Yndurain, Nucl. Phys. B153 (1979) 161;

A. Gonzalez-Arroyo and C. Lopez, Nucl. Phys. B166 (1980) 429.

2. J.A.M. Vermaseren, Int. J. Mod. Phys. A14 (1999) 2037.

3. J. Blümlein and S. Kurth, DESY 97-160, hep-ph/9708388; Phys. Rev. D60 (1999) 014018.

4. L. Schwartz, Théorie des Distributions (Hermann and Cie, Paris, 1951), Vols. I and II;

V.S. Vladimirov, Gleichungen der Mathematischen Physik, (DVW, Berlin, 1972);

K. Yoshida, Functional Analysis (Springer, Berlin, 1978).

5. E. Remiddi and J. Vermaseren, Int. J. Mod. Phys. A15 (2000) 725.

6. N. Nielsen, Nova Acta Leopold. XC, 121 (1909);

S. Kölbig, Siam J. Math. Anal. 17 (1986) 1232.

7. L. Lewin, Dilogarithms and Associated Functions (Mac-Donald, London, 1958); Polylogarithms and Associated Functions, (North Holland, New York, 1981);

A. Devoto and D.W. Duke, Riv. Nuovo Cim. 7 N6 (1984) 1.

8. L. Euler, Novi Comm. Acad. Sci. Petropol. 20 (1775) 140;

D. Zagier, First European Congress of Mathematics, (Birkhäuser, Boston, 1994), Vol.II, p. 497.

9. S. Moch, P. Uwer, and S. Weinzierl, J. Math. Phys. 43 (2002) 3363;

S. Weinzierl, Comput. Phys. Commun. 145 (2002) 357.

10. H. Hopf, Ann. of Math. 42 (1941) 22;

J. Milner and J. Moore, Ann. of Math. 81 (1965) 211;
M.E. Sweedler, Hopf Algebras, (Benjamin, New York, 1969).

11. C. Kassel, Quantum Groups, (Springer, Berlin, 1995).

12. A.B. Goncharov, Math. Res. Lett. 5 (1998) 497,

http://www . math . uiuc.edu/K-Theory/0297.

13. M. Waldschmidt, J. Théor. Nombres Bordeaux 12 (2000) 581; Multiple polylogarithms, Lectures at the Institute of Math. Sciences (Chennai, Nov. 2000); updated version http://www.math.jussieu.fr/ ${ }^{\sim m i w /}$ articles/ps/mpl.ps;

V.V. Zudilin, Uspekhi Mat. Nauk (Russian Math. Surveys) 58 (2003) 3.

14. W.L van Neerven and E.B. Zijlstra, Nucl. Phys. B382 (1992) 11.

15. S.A. Larin, T. van Ritbergen, and J.A.M. Vermaseren, Nucl. Phys. B427 (1994) 41;

S.A. Larin, P. Nogueira, T. van Ritbergen, and J.A.M. Vermaseren Nucl. Phys. B492 (1997) 338;

A. Retey and J.A.M. Vermaseren, Nucl. Phys. B604 (2001) 281.

16. J. Blümlein, Comput. Phys. Commun. 159 (2004) 19.

17. J. Blümlein, in preparation.

18. J. Blümlein and S. Moch, in preparation.

19. J. Blümlein, Comput. Phys. Commun. 133 (2000) 76 .

20. J. Blümlein and A. Vogt, Phys. Rev. D57 (1998) 1; D58 (1998) 014020.

21. C. Reutenauer, Free Lie Algebras, (Calendron Press, Oxford, 1993).

22. D.E. Radford, J. Algebra, 58 (1979) 432.

23. E. Witt, Journ. Reine und Angew. Mathematik, 177 (1937) 152; Math. Zeitschr. 64 (1956) 195.

24. M.E. Hoffman, J. Algebra, 194 (1997) 477.

25. D.J. Broadhurst and D. Kreimer, Phys. Lett. B393 (1997) 403. 